\title{
Reading Aloud in Human-Computer Interaction: How Spatial Distribution of Digital Text Units at an Interactive Tabletop Contributes to the Participants' Shared Understanding
}

\author{
Svenja Heuser ${ }^{(凶)}$, Béatrice Arend, and Patrick Sunnen \\ University of Luxembourg, Porte des Sciences 11, 4366 Esch-sur-Alzette, Luxembourg \\ \{svenja.heuser, beatrice.arend, patrick.sunnen\}@uni.lu
}

\begin{abstract}
This paper is concerned with how the spatial distribution of written informings in a serious game activity at an interactive tabletop (ITT) induces participants to read aloud interactionally relevant information to each other in the process of co-constructing a shared understanding.

Engaging in an unfamiliar game activity, the participants are all equally dependent on written informings from the interface that serve as a game manual and provide crucial information for jointly achieving the game task(s). When it comes to making use of these written informings, we find the participants to read them aloud, making them accountable within the group.

Our findings from multimodal video analysis of two reading-aloud cases suggest that the written informing's directionality and distribution (here, either designed as 'distributed' or 'shared' among the interface) regulate the participants' access to information. And that participants who cannot visually access the information they are interested in reading (aloud) co-organize fine-grained joint successive actions build on and actualized by read-aloud utterances. These joint actions allow them to align their orientation and share their understanding of game activity-relevant content.
\end{abstract}

Keywords: Reading aloud $\cdot$ Interactive tabletop $\cdot$ Collaboration $\cdot$ Conversation analysis $\cdot$ Interface design

\section{Introduction}

This paper addresses how participants mutually co-construct a shared understanding [1, 2] of an unfamiliar collaborative game activity they are engaging in (Fig. 1). The focus is on how they rely on written informings [see e.g., 3] displayed on the interface. These informings, mediated by an interactive tabletop (ITT, see Fig. 2) [4, 5], are designed to serve as a kind of game manual, to offer guidance and to provide the participants with crucial information for a gradual and joint familiarization with the ITT-activity. The collaborative activity is new to the participants and designed in a way that they all have to rely on these informings in order to jointly achieve several game task(s). 


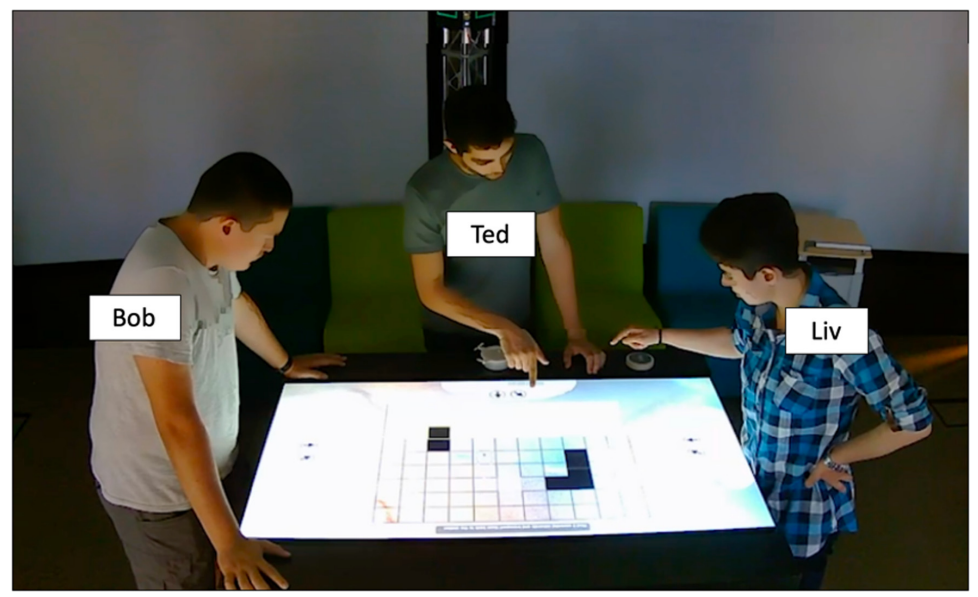

Fig. 1. Three participants (For the purpose of data protection, we edited the figures) engaging in 'Orbitia', each positioned at a referent control station. Together, they form a space mining crew, utilizing their control stations to steer a mining rover across the interface, collect essential minerals while avoiding damages and being economical with the resources provided.

Throughout our video corpus ${ }^{1}$, participants recurrently read aloud, allowing them to jointly and publicly share newly introduced information that is provided by the ITTsystem. In order for this new information to become part of a shared and intertwined understanding among the participants, it is related to what was already made sense of by then. In this process, it must be assessed and agreed upon to then form the basis of the group's joint next actions [2]. The way how participants use these read-aloud actions, as one way to share information and contribute to the group's co-construction of a shared understanding, is also 'influenced' by the design of the written informings on the ITTinterface (the spatial distribution and directionality of written informings, see Sect. 2). We shall see that the directionality of the digital text units regulates the participants' access to information. The same applies to the ratio of proximity and distance between both, the written informing on the interface (that is to be read aloud) and the participant (who aims to read it aloud).

In particular, by analyzing video data of multiparty game interaction at the ITT, we will show how participants use these written informings from the interface for producing reading-aloud accounts that facilitate shared understanding of the game activity in situ.

Relying on a multimodal conversation analytic approach [7, 8], we will present two cases of participants' reading alouds, one from a designated 'shared' written informing (information designed to be equally accessible to each participant) and one from designated 'distributed' written informings (information designed to provide privileged access to a particular player role by compartmentalizing it, see Sect. 2). At the end, we

\footnotetext{
${ }^{1}$ The above-mentioned video recordings are derived from the 'ORBIT' project (acronym for 'Overcoming Breakdowns in Teams with Interactive Tabletops') [6]. The research conducted especially on 'reading aloud' is part of the first author's dissertation project.
} 
will point to some practical design implications that become relevant with regard to our analysis.

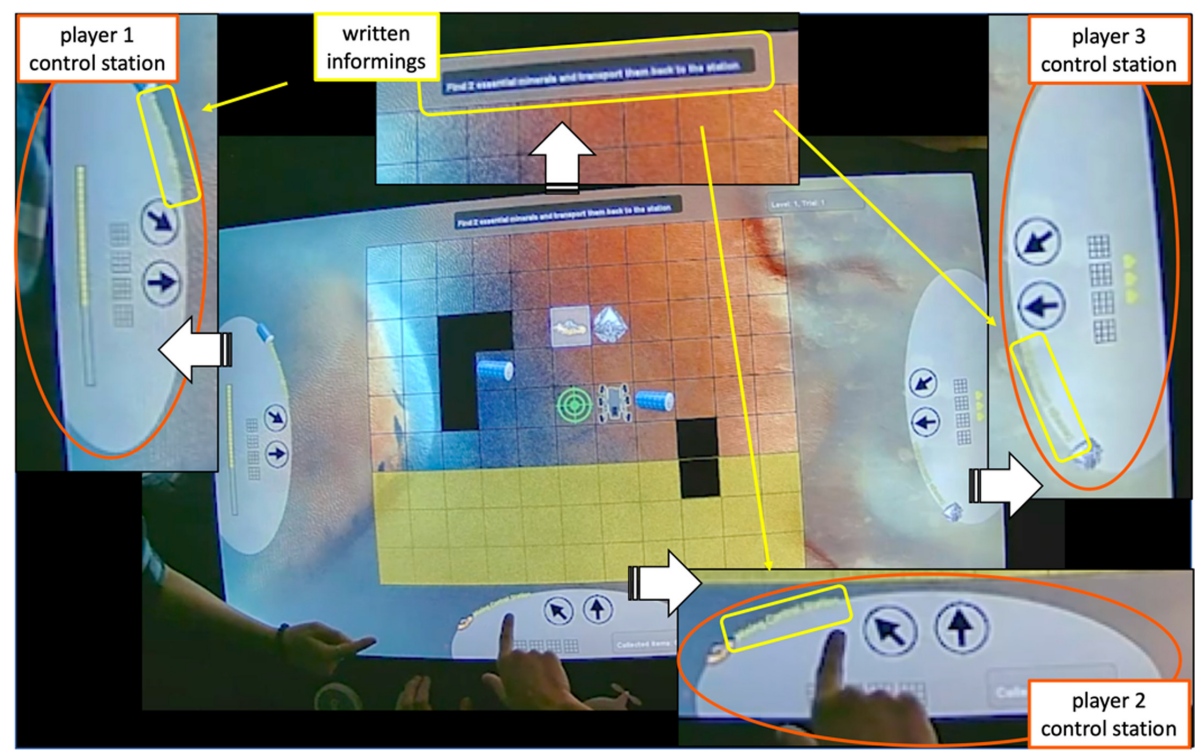

Fig. 2. Game world 'Orbitia', showing a planetary surface with three player 'control stations' (orange) (equipped with e.g., each two steering controls to maneuver the rover), a central grid and written informings (yellow). (Color figure online)

\section{Interface Design}

In the multiparty interaction at our ITT interface Orbitia, participants read aloud written informings mediated by the interface. In the here discussed excerpts, participants are all equally dependent on related information in order to jointly achieve the game task(s). As mentioned above, the written informings on the interface (each mostly about one to three sentences long) provide crucial information, serving as a game manual and offering guidance throughout the activity.

Looking more closely at research on text material being vocalized in participants' reading-aloud actions in game activity contexts, studies show that these so-called ingame texts [3] provide valuable resources for the players' joint goal orientation within the game activity [9]. It can also be found that the way they are located, formulated [10] (and translated [11]) influences the degree of supportiveness for the activity and the way the participants turn towards these written informings and similar activity-relevant information [12].

By design, the written informings displayed on the interface are either designed as 'distributed' or 'shared' among the participants (Fig. 3). Shared informings can be found in the large rectangular space of the interface that is represented as the planet's surface: 


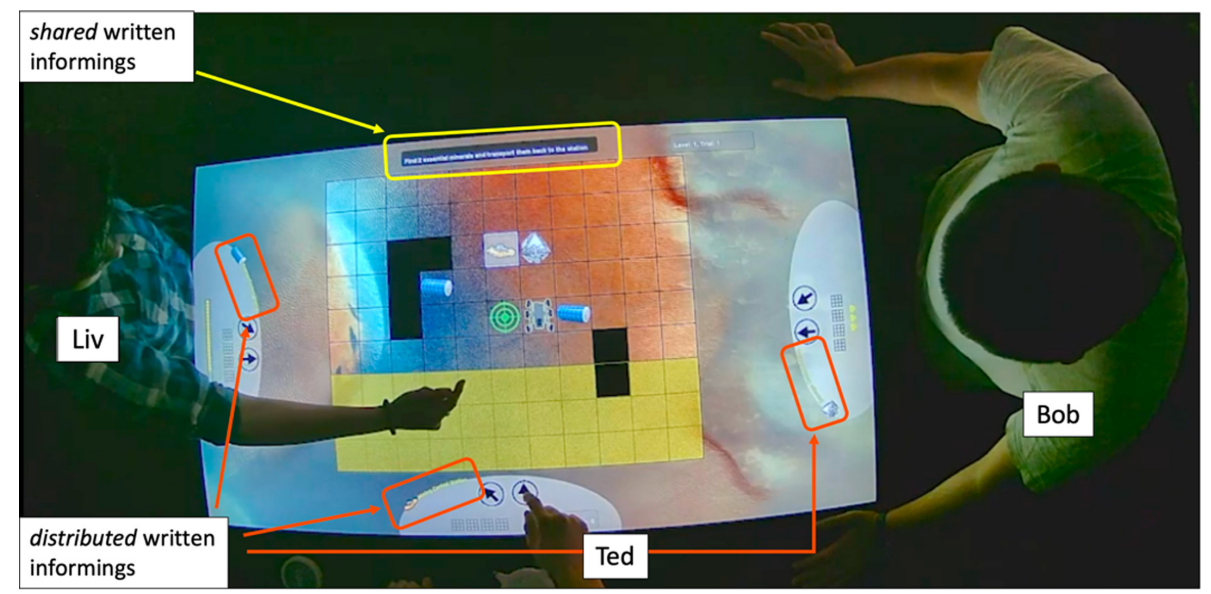

Fig. 3. In different areas of the interface, Orbitia provides written informings as 'shared' (e.g., on top, yellow) resp. 'distributed' (e.g., in each control station, orange). (Color figure online)

here either at the 'top' of the interface, visualized in a text box (see Fig. 3, e.g., 'Find two minerals and transport them back to the station.'), or others, related to the ITT's two tangible widgets ${ }^{2}$, displayed in a corona around the handle (e.g., 'drone' widget, see Fig. 4 and footnote 3: 'This is your drone. Move it on the grid and the number of hidden items will be displayed. Press the button to see them (4 attempts). This information will be sent to your control stations.'). Distributed informings can be found in the personal control stations of each player's position at the ITT. They are either permanently visible (e.g., as control station's labels, see Fig. 2, e.g., 'Mining Control Station') or visualized by the participants' activation. Besides the above-mentioned permanent written informings, all other informings need to be activated (respectively requested) by touching the referent digital buttons.

Next to the above-mentioned considerations concerning the distribution of written informings, also the mere size and shape of the tabletop interface and the designated participant positioning at the three sides (see Fig. 1 and 3) do come with certain restrictions in terms of directionality and visibility of the written informings when it comes to reading - or even more critical - reading aloud. Therefore, the directionality of graphic structures such as written informings must be carefully considered in the design process. The design approach of the above-shown interface was to provide for the shared written informings to be as directionless as possible, so that all participants had more or less equal visual accessibility for reading them. However, the more tilted (the closer to a

${ }^{2}$ In this paper, we will only focus on one of the two tangible widgets: the 'drone' tangible. As soon as the other tangible widget ('info' tangible) is inserted on the ITT, the interface system offers touchable 'info' icons at specific points across the interface (for requesting the related written informings). The 'info' tangible is also needed (on the table) for the other tangible widget ('drone' tangible) to display its attached written informing. In the subsequent version of Orbitia (described in [13]) we removed the info-widget and replaced it with distributed touch info-buttons. 


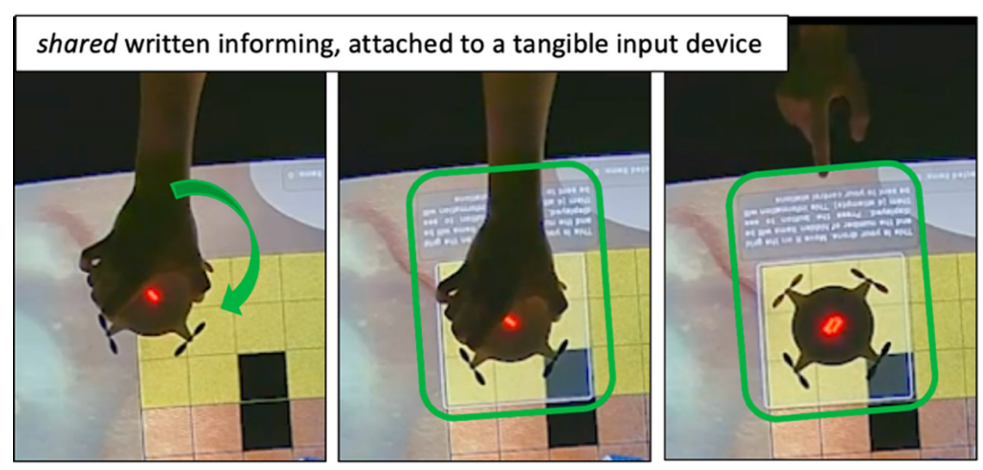

Fig. 4. The drone widget with a 'shared' text box attached. The latter is activated resp. visualized by the tangible's insertion onto the ITT surface.

180-degree rotation), the harder respectively the more strenuous it is to read them ${ }^{3}$. In contrast, distributed written informings (locally allocated according to each player's role in the game activity) should not be too easily accessible for other players in order for the informational asymmetry to induce collaborative exchange within the group [13]. Therefore, distributed informings face the respective individual player (and reader, see Fig. 3), shared informings however are either positioned on top of the interface (Fig. 3) or are attached to the mobile tangible widgets that can be positioned as required for a more convenient joint access (Fig. 4).

\section{Reading Aloud}

We consider reading aloud as a participants' practice of verbalizing resp. reading (parts of) written informings from the interface in a hearable voice, and by that, making this information mutually accessible within the group.

So far, the research on reading aloud in general is mainly conducted within a linguistic or a pedagogical framework and based on controlled speech data. E.g., these studies investigate recordings of instructed speakers reading aloud specific passages of a text repeatedly $[14,15]$ or audio recordings from telephone conversations [16]. In fact, only a few studies more closely deal with naturally occurring interactional phenomena in reading practices in general, and even less with read-aloud-speech in particular. All in all, the reading-aloud practice has only been investigated by a few scientists from a conversation analytic perspective. Swerts \& Geluykens [17] have examined speakers' prosodic performances in reading-aloud utterances, functioning as markers of information units, informing recipients about discourse boundaries. Koole [18] found that in classroom contexts reading aloud is often used by the student to localize a problematic passage in a written text that might have not yet been understood correctly.

\footnotetext{
${ }^{3}$ In a following version, the attached box with the written informing itself rotates with the rotation of the tangible widget. This is expected to facilitate the visual accessibility to the text from all sites of the interface.
} 
We find our participants incrementally producing delicately concerted multimodal read-aloud accounts for their understanding on how the game activity works and how they should proceed. In this process, they couple informational entities, they find on the interface (e.g., a written informing: 'Here is [...] your mining rover. Tap on the arrows to steer it.'), with corresponding touchable icons (e.g., two steering arrows at each participant's control station) and possible referent functions (e.g., maneuvering a mining rover across the grid to collect essential minerals).

So, the participants make their noticing of the written informings accessible for the other participants' assessment. The agreed upon understanding remains valid until new information is introduced that alters this understanding, building the basis for any joint next actions in this very situational context.

\section{Inducing Shared Understanding}

In order for the participants to collaboratively engage in the game activity, they need to interdependently and reciprocally develop and maintain a shared understanding of what the activity's goal is and of what they need to do as a group to achieve that common goal.

In particular, it is a continuously and 'jointly building up [of] a reciprocal understanding of each other's resources' [2: p. 3] that ideally results in a shared understanding among the group members [19]. Therefore, participants need to jointly figure out 'how they are going to work together, [...] with whom they are working' [20: p. 35], and, especially in a human-computer interaction context, 'how they should use [the] technology' [20: p. 35]. In this light, participants of our game activity need to make use and sense of multiple resources, create a joint focus, work interdependently, exchange ideas and information, and by that co-construct shared understanding of the game's task(s) and goal(s) [see 19].

More specifically, we aimed on creating an environment where the participants need to exchange on the resources provided. For that purpose, Orbitia provides three player roles (each player being in charge of either 'damage', 'mining' or 'energy' control, see Sect. 6.1, Fig. 5) with specific functions and responsibilities for the participants to take (over) within the game scenario. Additionally, multiple essential resources like written informings are made accessible in different ways. They are designed to be either as equally 'shared' as possible, or locally 'distributed' among the participants across the interface according to their roles in the game [13]. The above-mentioned design implementations create an asymmetry of knowledge among the participants that aims at inducing collaboration within the group. In the effort of understanding the rules and goals of the joint game activity, the participants need to articulate, piece together, discuss and evaluate on this complementary information. Exactly these processes can provide them with deeper and mutual insights into their roles as resources, and at the same time contribute to co-constructing, adjusting and maintaining a shared understanding.

Our focus of analysis is on "how knowledge is invoked and deployed as part of societal member's practical concerns" [1: p. 76]. In that sense, we observe (and understand) shared understanding as a mutual interactional accomplishment which dynamics depend on the fine-grained and constant reciprocal displaying of one's own and the monitoring 
of the other's understanding of the situation. It is an accomplishment that has to be jointly checked upon to be maintained over time.

\section{Method and Data}

The interface system was designed in the context of an interdisciplinary research project 'ORBIT' [6], bringing together researchers from social and computer sciences. Several user studies were conducted so far. The video corpus shows each three participants engaging in the game activity Orbitia in the lab facilities of a research institution. The analysis in this paper considers two read-aloud cases from one participant group. So far, the video recordings of three participant groups ( $95 \mathrm{~min}$.) have been scrutinized for the phenomenon in question here.

By relying on a conversation analytic approach $[7,21]$ in its multimodal understanding [8, 22], we shall examine the fine-tuned coordination of the interactional exchange between the participants' group and the ITT, and in more detail, one particular interactional practice for achieving shared understanding, which is reading aloud.

CA includes examining interactional phenomena, detecting procedures that constitute human social interaction working on case analysis and investigating the phenomenon across a corpus. Furthermore, CA informed analysis focuses on the participant's observable actions captured in video recordings. This research approach works on the analytical reconstruction of the participants' perspective and orientation to the specific context, here of human-computer interaction in situ.

Here, we can show how three participants jointly discover and mutually co-construct a shared understanding of game relevant features and challenges by reading aloud written informings. We find that co-constructing and maintaining shared understanding of both, previous and next actions, builds upon and is part of complex interactional processes. Making one's own understanding accountable and monitoring respectively assessing the other's responses is essential to achieving shared understanding. Here, reading aloud written informings from the ITT is a common way of how participants make text information from the interface accountable and include it into the ongoing interaction. The two cases presented in this paper will show how participants come to a shared understanding, gradually co-organizing their inter-exchange of information by reading aloud.

\section{Case Analyses}

We present two cases and point out, how reading aloud written informings from parts of the interface that may not be equally visually accessible for every participant is interactionally realized. In particular, the first case will show how the participants' reading aloud in the process of coming to a mutually shared understanding of their respective player positions and roles in the game activity is co-organized when coping with designated distributed written informings (see case I). Whereas the second case will show how their reading aloud, working on a mutual understanding of the digital interface structure and its respective tangible drone widget is co-organized when coping with a designated shared written informing (case II). 
Additionally, it will be outlined how the written informings' distribution and directionality contribute to co-constructing shared understanding in the sequential unfolding of the game activity.

\subsection{Case I: Coping with 'Distributed' Written Informings}

Right at the beginning of their engagement with the game activity, the participants begin to explore the digital surface (icons, texts and buttons) they find on the interface's grid and at each player's control stations (Fig. 5). To make sense of the semiotic resources at hand, the participants share and discuss their findings.

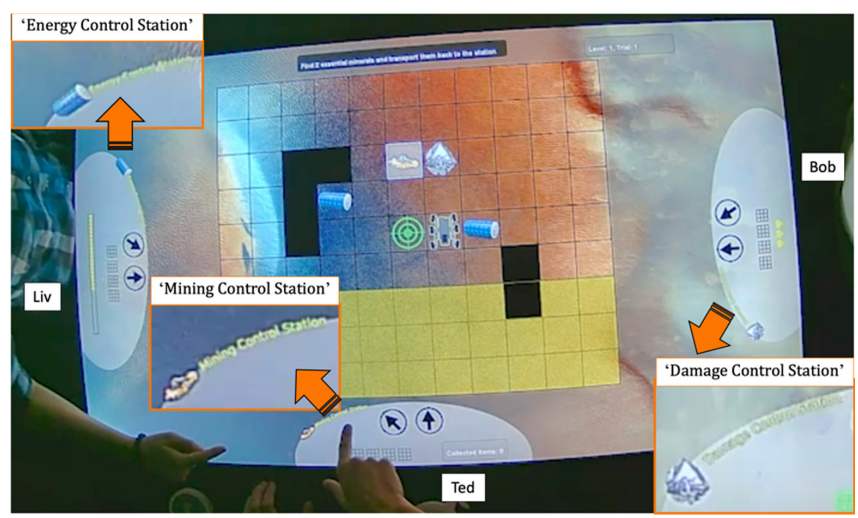

Fig. 5. ITT from top view, zooming in on the distributed written informings of each participants' control station (orange boxes). (Color figure online)

In Orbitia, each control station marks a position at the ITT and at the same time provides for a position and role for the player in the game activity. For that purpose, the stations are visually demarcated from the rest of the interface and labeled with the respective role allocation: 'Energy Control Station', 'Mining Control Station' and 'Damage Control Station' (Fig. 5).

In the following sequence ${ }^{4}$ Liv is focused on the possible steering functions. Each player is provided with two complementary steering directions in her/his control station, enabling them to jointly maneuver a rover over the planet's surface.

4 The transcript lines indicate the participant and the interactional resource that is transcribed: ver $=$ verbal conduct, ges $=$ gestural conduct, pos $=$ the participants' physical conduct in term of positioning movements. Additionally, the italics marked with an asterisk show the references between transcript lines and figures. 


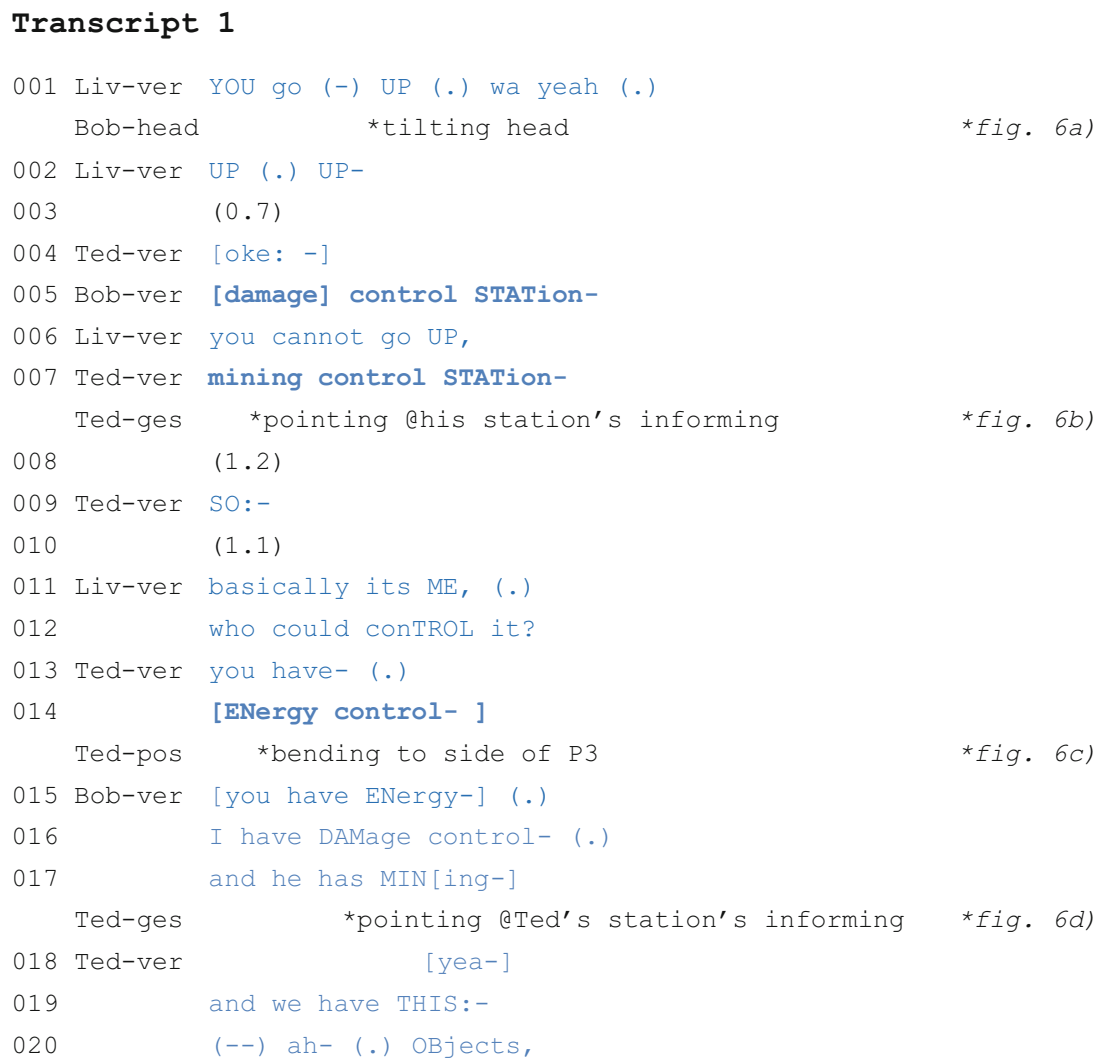

Liv, engaged into the steering functions, is instructing Ted ('you go up', transcript 1, 1. 1) in terms of using the directional arrows in front of him, verbalizing and (gesturally) demonstrating her understanding of 'up' (see Fig. 6a, 1. 1). His 'up' is her 'left', showing her already undertaken change of perspectives within her steering instruction elicited by the interface's design (in particular, by the above-mentioned distribution of steering resources). The instructed Ted presses both arrows without receiving visual feedback from the interface and as a response produces a stretched 'oke' (1. 4). Meanwhile Bob tilts his head, aligning with Ted's perspective onto the ITT interface next to him (1. 1, Fig. 6a), then returning to his upright home position at the table, visually orienting down onto his control station and reading aloud its label ('damage control station', 1. 5) which overlaps with Ted's current turn. Liv responds with a question referring to Ted's steering attempt ('you cannot go up', 1. 6) with a high rising intonation at the end and does not address Bob's (read aloud) account on his written station's label.

Now Ted reads aloud his control station's label ('mining control station', 1. 7) and simultaneously performs a pointing gesture onto the latter (Fig. 6b). With the vocalization of the second word he orients slightly to Liv's control station to his left (Fig. 6c), then to Bob's station to his right. In the course of about two seconds after Ted's read-aloud utterance, Liv visually orients to Ted's station, then to Bob's and then to her own. And so, do the others. Within the first and the second reading aloud utterance, each participant 

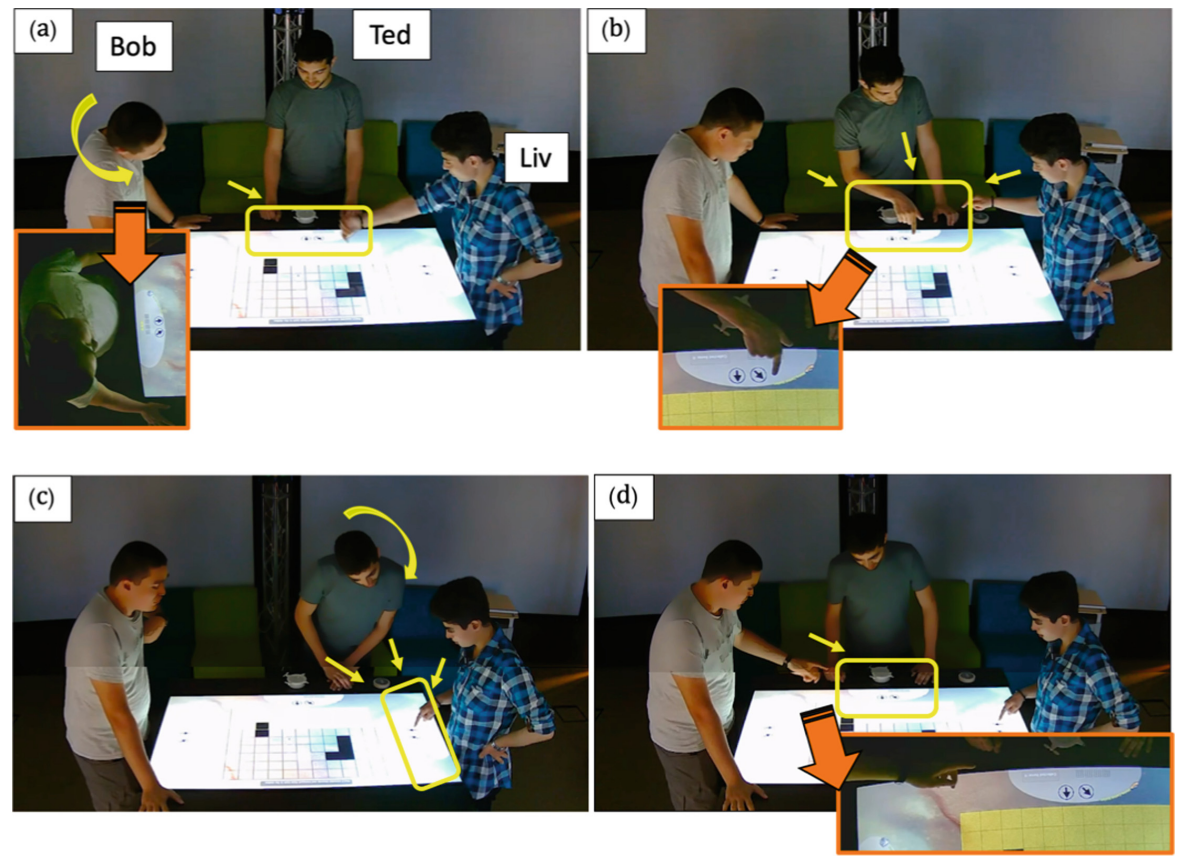

Fig. 6. a-d. ITT-areas made relevant by specific deployment of bodily resources (e.g., finger pointing, bending over torso) for achieving joint visual orientation onto the relevant area on the interface (yellow borders and arrows). (Color figure online)

visually orients at least once to each other participant's control station, readjusting their heads and gazes for the purpose of getting (better) visual access, before orienting down again to their own control stations right in front of them.

Thereafter Ted expands his previous read-aloud turn (1. 7) with a stressed and stretched pivotal marker ('so', 1. 9) [see e.g., 24 or 25]. When no one jumps in offering relevant information, Liv makes an account for her understanding of the way (at least) the steering is handled ('it's me who could control it', 1. 11-12). She does that with intonation rising high at the ends of both sentences, prosodically realizing a question for clarification. Thereby she keeps on following her exploratory interest of how the steering is conducted, while at the same time responding to the read-aloud accounts of Bob and Ted. As the other two utter respectively share that they are in charge of each 'damage' and 'minerals', she states her understanding of being responsible for the steering 'control' (1. 11-12), and that the two others cannot assist her with that.

After Liv states her understanding of her role in this game activity, Bob and Ted both orient towards her panel: Bob on the opposite side making himself taller, Ted on the connected side leaning down and to her station (Fig. 6c). He first utters 'you have' (1.13) and then pauses while bending over to Liv's panel even more, tilting his head so that he can more easily access respectively read what is written on the top of her control station. This direct response to Liv's question raised is followed by a pause, giving Ted time to fully bend over (Fig. 6c) and produce the reading (aloud) of the two words ('energy 
control', 1. 14). Ted's response is designed for Liv to inform her about his understanding of what she is responsible of ('you have energy control', 1. 13-14). He is, on the one hand, responding to her question raised if her task in this game and player configuration was to control the steering all by herself ('it's me who could control it', 1. 11-12), and, on the other hand, providing her with the information about what her personal station's label is actually saying. His account is a paraphrasing utterance, incorporating a readaloud information into the syntax of his utterance (first part: Ted's own speech material, adjusted to address liv: 'you have', and second part: a read-aloud utterance from Liv's control station: 'energy control'). Within the pause of Ted's above-described utterance, Bob initiates an utterance himself, overlapping with Ted's read-aloud part (1. 14). In particular, not only the realization of both utterances slightly overlaps, but also their content is partly congruent: Bob and Ted both utter 'you have energy' (1. 13-15).

Bob verbalizes (1) 'you have energy' (1. 15), looking onto Liv's side, (2) 'I have damage control' (1. 16), looking down onto his panel, (3) 'and he has mining' (1. 17), orienting slightly to his left towards Ted's panel. The last part is accompanied by a deictic gesture into the direction of Ted's station, reaching its gestural peak with the uttering of 'he' (referred to Ted, 1. 17, Fig. 6d). Each of these three clauses is constituted of a) the personal pronoun of the player that is referred to (from Bob's personal perspective, designed for Liv's understanding): 'you' refers to Liv, 'I' refers to himself, 'he' to Ted. Then b) the grammatically referent verb form of possession and c) the one noun of each station's label that differentiates the three control stations from one another ('energy' for Liv, 'damage' for himself and 'mining' for Ted). He can extract that from the previous reading and reading aloud actions and incorporates it into a consecutive and precise enumeration.

For the purpose of making his very own understanding accountable and trying to make the others understand, Bob is occupying his own and each of the other participants' perspectives, positions and roles at the ITT. He sums up what the other stations are saying (and meaning) in relation to each other, working on a coupled understanding on how the relations of positions, functions, tasks and responsibilities are intertwined among the participants and across the interface.

Also, Ted's and Liv's visual orientation follows Bob's enumeration of station labels, directing their visual orientation to the addressed stations, one after the other. Bob's expanded summary of the station's labels (that Ted initiated with his read aloud) is responded to with Ted's positive assessment ('yea', 1. 18). Ted then extends this utterance, enumerating and grabbing the two tangible widgets they have been provided with, introducing further unfamiliar things ('this objects', 1. 19-20) for the group to jointly make sense of. No matter what, they proceed with initiating joint next actions, and among others, discussing about the location of the rover (which is to be steered jointly across the interface).

Findings Case I: In order for the group to jointly figure out how to proceed with relevant next actions, the participants systematically access and share information from three distributed written informings from each participants' control station that are all informationally coherent but not congruent. With the help of read-aloud accounts (sharing the specific information and its location, making it accountable for everyone to both see and hear), the participants build the basis for the group's involvement with and exchange 
about the new verbally introduced information. Bob makes accountable that he understands himself and Ted already being 'on the same page' and that they now both make the effort of also getting Liv on board to finally reach some shared understanding. Their subsequent synoptic utterances (performed by two participants, Ted and Bob, 1. 13-17), precisely designed for its recipients, root on the information recently introduced by the participant's read alouds (1.5 and 7) and are therefore mutually dependent.

\subsection{Case II: Coping with a 'Shared' Written Informing}

After coming to the shared understanding of each participant's allocated role in the game activity (see case I), they now commence inspecting and manipulating the two tangible widgets ('info' and 'drone' tangible). After the participants inserted one widget ('info') onto the ITT surface, they are now inserting the second widget ('drone', see Fig. 7a). Once the tabletop system detects the widget in proximity of the surface a highlighted corona is visualized around the handle together with a text (see Sect. 2, Fig. 4 and Sect. 6.2, Fig. 7a-b) that is designed to be used as a shared written informing for all participants to access equally: 'This is your drone. Move it on the grid and the number of hidden items will be displayed. Press the button to see them (4 attempts). This information will be sent to your control stations.' (see Fig. 7a and b)

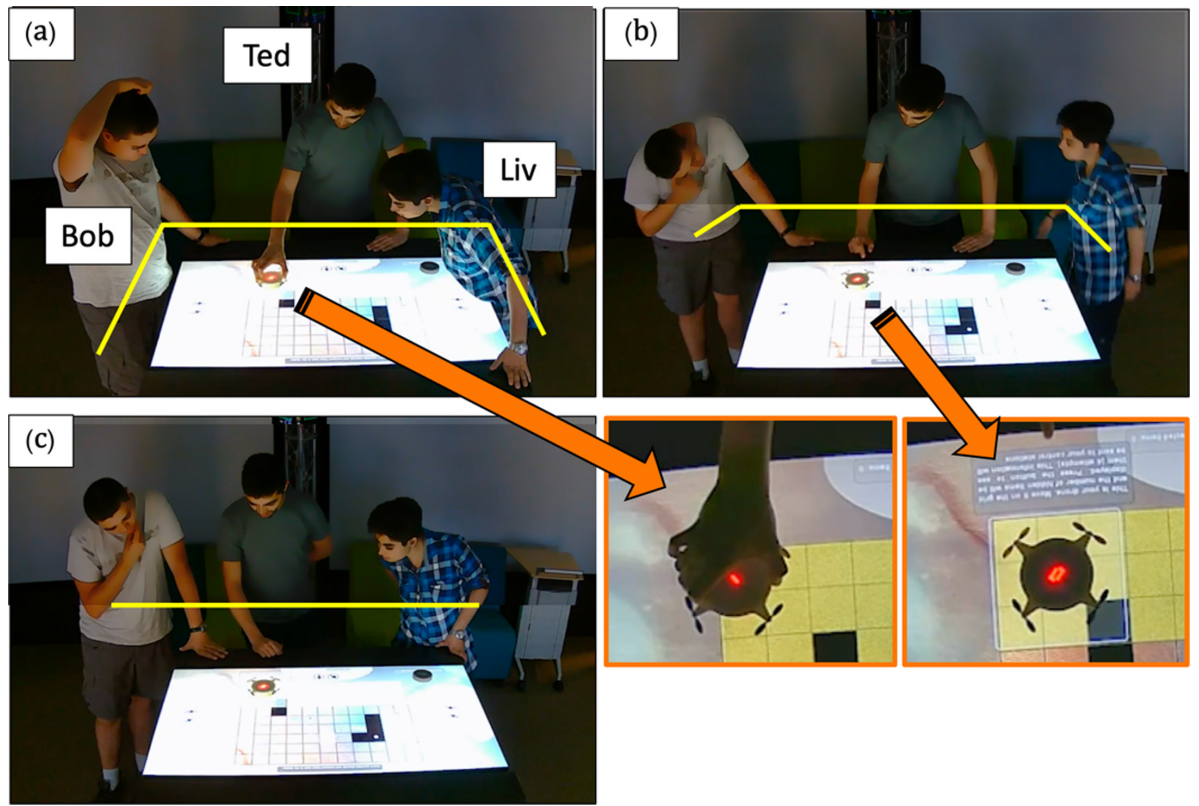

Fig. 7. a-c. Participants' physical (re-)positioning movements alongside the ITT (yellow lines) after insertion of the drone tangible that activates the written informing (orange boxes). (Color figure online) 
In the following sequence ${ }^{5}$ the visualization of a shared written informing elicits the participants' alignment of orientation onto the text unit and its reading aloud.

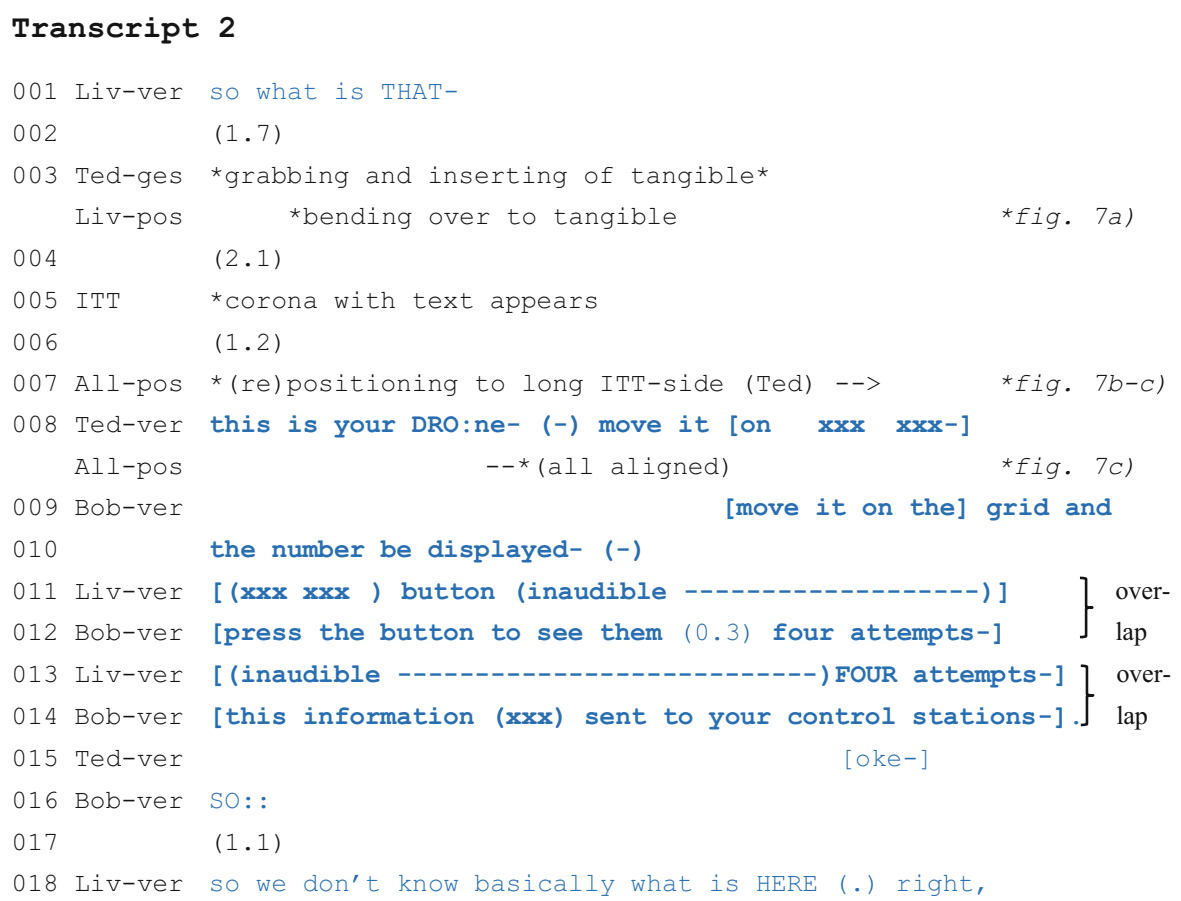

Showing her interest in the exploration of the above-described drone tangible, Liv orients to it, (first verbally: 'so what is that', transcript 2,1. 1), making it accountable and interactionally relevant for the others' next actions, as we shall see in the following. Ted then grabs the drone-widget from the side of the table and moves it over the interface in front of him (1. 3), while Liv bends over the tabletop to cover a maximum distance to get clear sight (then physically: 1. 3, Fig. 7a). The written informing appears (1. 5). It is directed towards Ted so that he can easily get visual access, while the other two have a more lateral view (see Fig. 7a).

Bob, who's sight onto the written informing is blocked by Ted's arm, bends down strongly (Fig. 7b) until Ted retracts his arm, steps back slightly and makes space for the other two participants (Bob, Liv) to initiate (re)positioning movements towards the long side of the table (Ted's side; see Fig. 7a-c, 1. 7). Before they are all aligned in one row (Fig. 7c), Ted, who is already in visual proximity of the text initiates a readaloud utterance (1. 8, see also Fig. 8). He starts with the text's first sentence and his first contribution in the process of building a shared understanding. When Bob finds his place alongside Ted, he sets in reading aloud (1. 9, Fig. 8) with the beginning of the second sentence of both, the written informing and Ted's reading-aloud utterance. His

5 The transcript lines also indicate the ITT's conduct: ITT = visual feedback of the interface system. In addition, the ' $x$ 's' mark inaudible verbal conduct of one to two syllables. Longer inaudible parts of utterances are simply marked 'inaudible'. 
accountable sharing of information overlaps with Ted's read-aloud utterance (1. 8 and 9). Ted as a response cancels his reading aloud in the middle of the second sentence. So, only a few syllables after Bob starts to speak simultaneously. Only shortly after Liv's arrival at the long side of the table (Fig. 7c) and Ted's turn-termination, Liv initiates a read-aloud utterance herself (1. 11, see also Fig. 8). She sets in with both, the third sentence of the written informing and Bob's utterance. The latter however is overlapping with Bob's current read aloud. The two of them simultaneously come to the completion of their reading aloud of (parts of) the rest of the written informing (1. 13 and 14, Fig. 8). Both, Bob's and Liv's read alouds are syntactically elliptic and not completely congruent to the written informing's text. Liv's read aloud is in addition not vocalized clearly for most parts, prosodically designed in a low-voiced and by tendency mumbled manner (which would make it hard for the recipients to utilize her utterance as an informing account). Nevertheless, the other participants treat it as accounts of engagement. Ted anticipates and treats the completion of the joint read-aloud action of the other two participants and assesses the new information input with a pivotal marker 'oke' (1. 15). Liv thereafter sums up her understanding of where the drone tangible (they just manipulated and read about) should be deployed (enabling them to see 'what is here', 1. 18). They then (not transcribed here) will commence to explore the interface together on the basis of their only just discovered and shared information, and of course their understanding.

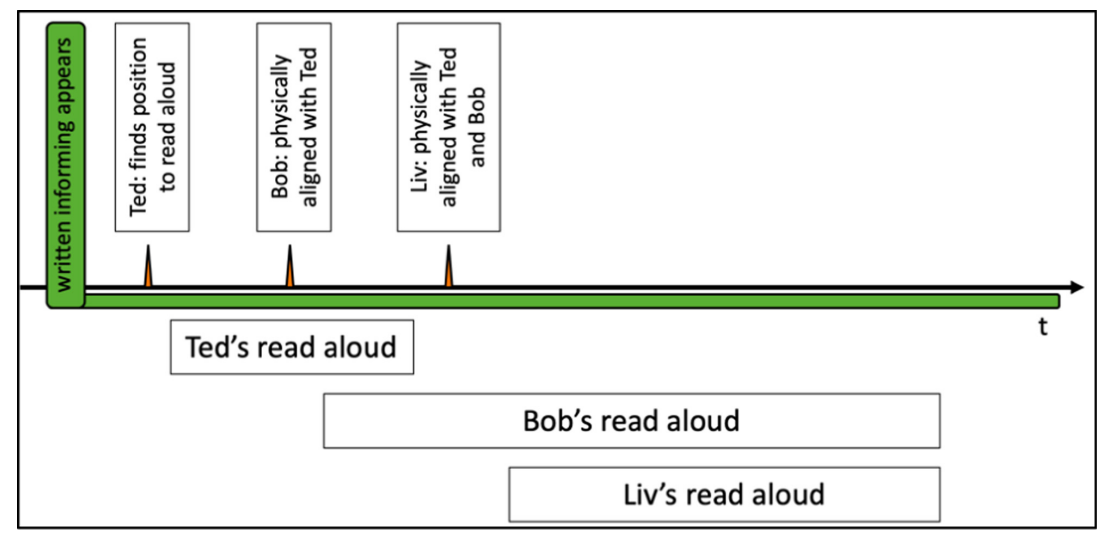

Fig. 8. Scheme of the sequential unfolding of participants' actions: (re) positioning movements in relation to the production of read aloud utterances and their overlaps.

As described above, the participants co-organize their reading-aloud utterances in a fluent taking over and smooth blending in, producing a successive chaining of actions that facilitates one joint read-aloud action. In fact, they all perceive not only the other's joint bodily orientation onto the same text entity, but also the(ir) referent joint verbal read-aloud action. There seems to be no necessity of reading it (aloud) again, even though their utterances (themselves, and even more so jointly) might not have been perfectly acoustically perceivable to one another, word for word. Nevertheless, they mutually display their joint engagement regarding the relevance of the read-aloud written informings as well as their joint alignment regarding the practice to interactionally handle 
written informings. The participants' accomplishment of the above-described joint readaloud action is directly followed by positive assessment and by the inclusion of the introduced information into the organization of joint next actions.

Findings Case II: In order to visually access the shared written informing in the tangible's corona simultaneously, the participants undertake interactional work by physically (re)positioning their bodies at the ITT interface and verbally orchestrating a continuous and jointly (at least) hearable reading-aloud action. By doing so, they all equally and simultaneously make their engagement into the current activity interactionally accountable. Here, participants prosodically and temporally couple the text and its reading-aloud in some rhythmic drive, where group members 'jump in' at the right spot at the right time (instead of starting new at the text's beginning) to maintain the progressing multilayered interplay of the joint read-aloud action in the process of developing shared understanding.

\section{Conclusion}

Due to the interface's design that presets the location (and accessibility) of written informings, in the process of exploring the 'distributed' written informings in their control stations (case I), Bob and Ted both share their understanding of each participants' different position and role by reading (them) aloud. To also include Liv into this process, they successively produce a complex series of utterances, sharing and aligning all participants' perspectives and referent roles, made accessible through their reading aloud of the relevant written informings. The latter are each directed to the referent stations' player position, enabling direct visual access for the one participant at that very station while at the same time restricting but not hindering the visual access from other stations' positions at the ITT. Due to this specific three-parted spatial distribution, the participants solve the interactional challenge of information (in)accessibility by taking each other's perspectives and publicly sharing their accumulated understanding(s) of it.

Coping with the 'shared' written informing (case II), the participants reciprocally (both physically and verbally) adjust their joint actions in a different way. Here, the properties of the shared written informing (designed to be as equally accessible as possible for all participants), in particular the text's position (in arm's reach of the participant who operates the tangible widget) and directionality (in optimal angle for the latter to read) elicits them to all align their bodies and perspectives at this participant's side of the table and to jointly access all relevant information. Co-organizing a shared turn [see 26, 27] they manage to utilize the specific structures provided by the interface to temporarily align their perspectives and synchronize their actions. As a result, they can all jointly proceed with broadening, deepening and aligning their understanding of the situation on the basis of the new information. The way they jointly co-produce this read-aloud action, they assure that it was fully reciprocally accountable and equally perceivable, because each participant has both read it (aloud) and witnessed each of the other's to have read it (at least partly) aloud.

Both cases presented in this paper show the participants' fine-grained co-organization of joint successive actions when dealing with the interactional challenge of accessing 
relevant information on an interface, actualized and constructed by read-aloud utterances. These read-aloud's serve as a vehicle for the participants' mutually displaying and monitoring of the individual's as well as the other's understanding in the process of co-constructing a shared understanding within the group. Furthermore, the spatial distribution of text across the horizontal interface facilitates the participants' interactional effort for accomplishing an alignment of their multimodal resources and orientations. So, the participants jointly reach a shared understanding of game activity-specific content.

Based on the above-described observations on how participants in our game activity Orbitia cope with (the reading aloud of) differently distributed written informings on the interface we could see that these informings and their spatial distribution induce collaborative exchange among the participants.

Slightly different to our expectations (the designated shared written informings to be rather equally accessible for the participants, and the designated distributed written informings to provide rather privileged access for particular participants), the written informings, designed as shared and co-equally accessible (see case II) likewise required the group's joint adjustment actions for the purpose of reading them aloud (although of course in a different way). In a next iteration of the interface system, this designated 'shared' text attached to the drone tangible was fixed to the latter in a way that by participants' rotation of the tangible the text would rotate as well. It would be interesting for future analyses to investigate on how e.g., this design adjustment now facilitates the participants to access the drone's written informing and share it within the group.

Acknowledgements. We thank the Luxembourg National Research Fund (FNR) for funding the ORBIT project (Overcoming Breakdowns in Teams with Interactive Tabletops) under the CORE scheme and our project colleagues Valérie Maquil and Hoorieh Afkari from the Luxembourgish Institute of Science and Technology (LIST). We also thank the participants.

\section{References}

1. Arnseth, H.C., Ludvigsen, S., Wasson, B., Mørch, A.: Collaboration and problem solving in distributed collaborative learning. In: Dillenbourg, P., et al. (eds.) European Perspectives on Computer-Supported Collaborative Learning: Proceedings of Euro-CSCL 2001, pp. 75-82 (2001)

2. Arend, B., Heuser, S., Maquil, V., Afkari, H., Sunnen, P.: Being a space mining crew: how participants jointly discover their complementary resources while engaging into a serious game at an interactive tabletop (ITT). In: Gadille, M., Caraguel, V. (eds.) Building the New Millenium Skills for Project Management in Higher Education: How Virtual Worlds Can Help Teachers And Students?, EDULEARN20, 12th Annual International Conference on Education and New Learning Technologies (2020)

3. Evans, J.: Translating board games: multimodality and play. J. Specialised Transl. 20, 15-32 (2013)

4. Antle, A., Wise, A.: Getting down to details: using theories of cognition and learning to inform tangible user interface design. Interact. Comput. 25(1), 1-20 (2013)

5. Anastasiou, D., Maquil, V., Ras, E., Fal, M.: Design implications for a user study on a tangible tabletop. In: Proceedings of the 15th International Conference on Interaction Design and Children, pp. 499-505. ACM, New York (2016) 
6. Sunnen, P., Arend, B., Maquil, V.: ORBIT - overcoming breakdowns in teams with interactive tabletops. In: Kay, J., Luckin, R. (eds.), Rethinking Learning in the Digital Age: Making the Learning Sciences Count, 13th International Conference of the Learning Sciences (ICLS) 2018, London, UK, pp. 1459-1460 (2018)

7. Psathas, G.: Conversation Analysis: The Study of Talk-in-Interaction, vol. 35. Sage Publications, Thousand Oaks (1994)

8. Mondada, L.: The organization of concurrent courses of action in surgical demonstrations. In: Streeck, J., et al. (eds.). Embodied Interaction. Language and Body in the Material World, pp. 207-226. Cambridge University Press, Cambridge (2011)

9. Thorne, S.L., Fischer, I., Lu, X.: The semiotic ecology and linguistic complexity of an online game world. ReCALL 24(3), 279-301 (2012)

10. Mason, J.: Video games as technical communication ecology. Techn. Commun. Q. 22(3), 219-236 (2013)

11. Šiaučiūnè, V., Liubinienè, V.: Video game localization: the analysis of in-game texts. Stud. Lang. 19, 46-55 (2011)

12. Woodruff, A., Szymanski, M.H., Aoki, P.M., Hurst, A.: The conversational role of electronic guidebooks. In: Abowd, G.D., Brumitt, B., Shafer, S. (eds.) Ubicomp 2001: Ubiquitous Computing, UbiComp 2001, vol. 2201, pp. 187-208. Springer, Heidelberg (2001). https://doi.org/ 10.1007/3-540-45427-6_16

13. Sunnen, P., Arend, B., Heuser, S., Afkari, H., Maquil, V.: Developing an interactive tabletop mediated activity to induce collaboration by implementing design considerations based on cooperative learning principles. In: Stephanidis, C., Antona, M. (eds.) 22nd HCI International Conference 2020. Denmark, Springer, Copenhagen (2020). https://doi.org/10.1007/978-3030-50729-9_45

14. Thorsen, N.: Intonation and text in Standard Danish. Ann. Rep. Inst. Copenhagen 18, 185-242 (1984)

15. Lass, N.J., Lutz, D.R.: The consistency of temporal speech characteristics in a repetitive oral reading task. Lang. Speech 18(3), 227-235 (1975)

16. Local, J., Walker, G.: How phonetic features project more talk. J. Int. Phonetic Assoc. 42(3), 255-280 (2012)

17. Swerts, M., Geluykens, R.: Prosody as a marker of information flow in spoken discourse. Lang. Speech 37(1), 21-43 (1994)

18. Koole, T.: Teacher evaluations. Evaluating Cogn. Competences Interact. 225, 43 (2012)

19. Roschelle, J., Teasley, S.D.: The construction of shared knowledge in collaborative problem solving. In: O'Malley, C. (ed.) Computer Supported Collaborative Learning, NATO ASI Series, vol. 128, pp. 69-97. Springer, Berlin (1995). https://doi.org/10.1007/978-3-642-850 98-1_5

20. Mulder, I., Swaak, J., Kessels, J.: Assessing group learning and shared understanding in technology-mediated interaction. J. Educ. Technol. Soc. 5(1), 35-47 (2002)

21. Schegloff, E., Jefferson, G., Sacks, H.: A simplest systematics for the organization of turntaking for conversation. Language 50(4), 696-735 (1974)

22. Deppermann, A.: Multimodal interaction from a conversation analytic perspective. J. Pragmat. 46(1), 1-7 (2013)

23. Selting, M., Auer, P., Barth-Weingarten, P., et al.: A system for transcribing talk-in-interaction: GAT 2, translated and adapted for English by Couper-Kuhlen, E., Barth-Weingarten, P. (eds.) Gesprächsforschung - Online-Zeitschrift zur verbalen Interaktion 12, pp. 1-51 (2011)

24. Szczepek Reed, B., Reed, D., Haddon, E.: NOW or NOT NOW: Coordinating restarts in the pursuit of learnables in vocal master classes. Res. Lang. Soc. Interact. 46(1), 22-46 (2013)

25. Proske, N.: Zur Funktion und Klassifikation gesprächsorganisatorischer Imperative. In: Diskursmarker im Deutschen Reflexionen und Analysen, pp. 73-101. Verlag für Gesprächsforschung, Göttingen (2017) 
26. Lerner, G. H.: Turn-sharing. In: The Language of Turn and Sequence, pp. 225-256. Oxford University Press, New York (2002)

27. Pfänder, S., Couper-Kuhlen, E.: Turn-sharing revisited: an exploration of simultaneous speech in interactions between couples. J. Pragmat. 147, 22-48 (2019)

Open Access This chapter is licensed under the terms of the Creative Commons Attribution 4.0 International License (http://creativecommons.org/licenses/by/4.0/), which permits use, sharing, adaptation, distribution and reproduction in any medium or format, as long as you give appropriate credit to the original author(s) and the source, provide a link to the Creative Commons license and indicate if changes were made.

The images or other third party material in this chapter are included in the chapter's Creative Commons license, unless indicated otherwise in a credit line to the material. If material is not included in the chapter's Creative Commons license and your intended use is not permitted by statutory regulation or exceeds the permitted use, you will need to obtain permission directly from the copyright holder.

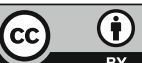

